\title{
Eficiência relativa de fontes de silício no controle de brusone nas folhas em arroz ${ }^{(1)}$
}

\author{
Rodrigo Fascin Berni(2) e Anne Sitarama Prabhu(3)
}

\begin{abstract}
Resumo - A adubação silicatada constitui uma das alternativas para diminuir o uso de fungicidas no controle da brusone em arroz. Foi realizado um experimento, durante 1999/2000 e repetido durante 2000/2001, com o objetivo de estudar a eficiência relativa de fontes de silício ( $\mathrm{Si}$ ) na redução da severidade da brusone nas folhas da cultivar Metica-1, em área de várzea. Os tratamentos foram três fontes de $\mathrm{Si}$ (silicato de alto forno; serpentinito, minério rico em $\mathrm{Si}$ e wollastonita, um metasilicato de cálcio natural), em cinco doses $\left(0,0,5,1,0,2,0\right.$ e 4,0 $\left.\mathrm{Mg} \mathrm{ha}^{-1}\right)$ sem tratamento das sementes ou com tratamento das sementes com o fungicida pyroquilon $200 \mathrm{~g}$ de i.a. por $100 \mathrm{~kg}$. A severidade da brusone diminuiu significativamente com o aumento de doses de silício. As relações entre a área sob curva de progresso da doença e as doses, tanto do silicato de alto forno quanto da wollastonita, foram lineares e negativas, com ou sem tratamento de sementes, em ambos os anos do experimento. Os resultados revelam o aumento da eficiência do tratamento das sementes com fungicida na redução da brusone nas folhas com a fertilização silicatada.
\end{abstract}

Termos para indexação: Oryza sativa, Pyricularia grisea, Magnaporthe grisea, método de cultivo.

\section{Relative efficiency of silicon sources on rice leaf blast control}

\begin{abstract}
Silicon fertilization of rice is one of the alternatives for reducing the use of fungicides in the control of rice blast. An experiment was conducted, during 1999/2000 and repeated in 2000/2001, in order to study the efficiency of sources of silicon (Si) to reduce leaf blast on rice cultivar Metica-1, cultivated in the low land. The treatments included three sources of Si (high furnace silicate slag, serpentinite ore rich in silicon and wollastonite ore, a native calcium metasilicate) at five doses $(0,0.5$, 1.0, 2.0 and $4.0 \mathrm{Mg} \mathrm{ha}^{-1}$ ), with and without fungicide treatment (pyroquilon $200 \mathrm{~g}$ a.i. per $100 \mathrm{~kg}$ of seed). The blast disease severity decreased with increase in Si doses. The relationship between area under disease progress curve and doses of silicate slag as well as wollastonite were linear and negative, with and without seed treatment. Furthermore, the results show the increased efficiency of seed fungicide treatment in reducing blast severity, with silicon fertilization.
\end{abstract}

Index terms: Oryza sativa, Pyricularia grisea, Magnaporthe grisea, cultural methods.

\section{Introdução}

A brusone, causada por Pyricularia grisea (Cooke) Sacc. [=Magnaporthe grisea (Herbert) Barr], é a principal enfermidade do arroz. Os motivos da alta ocorrência dessa enfermidade são a breve durabilidade da resistência das cultivares e a intensificação do cultivo de arroz inundado, especial-

\footnotetext{
(1) Aceito para publicação em 15 de outubro de 2002.

(2) Embrapa-Centro de Pesquisa Agroflorestal da Amazônia Ocidental, Rod. AM-010, Km 29, CEP 69011-970 Manaus, AM. E-mail: rodrigo@cpaa.embrapa.br

(3) Embrapa-Centro Nacional de Pesquisa de Arroz e Feijão, Caixa Postal 179, CEP 75375-000 Santo Antônio de Goiás, GO E-mail: prabhu@cnpaf.embrapa.br
}

mente em condições tropicais, como o Estado do Tocantins. As perdas na produtividade de grãos em decorrência da brusone, em condições de campo, variaram de $15 \%$ a $38 \%$ e de $37 \%$ a $44 \%$ nas cultivares precoces e de ciclo médio, respectivamente (Prabhu et al., 1986). Para cada $1 \%$ no aumento na severidade da brusone, ocorre a redução média de $2,7 \%$ e $1,5 \%$ nas cultivares de ciclo precoce e médio, respectivamente (Prabhu et al., 1989). As perdas provocadas pela brusone nas folhas são decorrência indireta da redução da área foliar fotossintetizante, do crescimento e desenvolvimento da planta (Goto, 1965).

O desenvolvimento de tecnologias para o manejo sustentável da brusone depende da disponibilidade de métodos alternativos de controle. O silício (Si) aumenta o crescimento e o desenvolvimento da plan- 
ta com correspondente acréscimo na produtividade, além de controlar várias enfermidades do arroz (Savant et al., 1997). O rendimento de grãos das cultivares de arroz de sequeiro aumentou de forma linear com a fertilização silicatada e foi correlacionado positivamente com os teores de $\mathrm{Si}$ e Ca no solo (Barbosa Filho et al., 2001). O desempenho do Si no aumento da resistência das plantas às diferentes enfermidades, inclusive à brusone em arroz, tem sido bem reconhecido (Datnoff et al., 1991, 1992, 1997; Osuma-Canizales et al., 1991; Winslow, 1992; Zambolin \& Ventura, 1993; Korndörfer et al., 1999).

Estudos pioneiros no Japão demonstraram que a aplicação de diversas fontes de $\mathrm{Si}$ em solos deficientes neste elemento reduziram a incidência e a severidade da brusone (Suzuki, 1965). O efeito residual da fertilização silicatada no controle da brusone também foi demonstrado em experimentos realizados no campo, na Flórida e na Colômbia (Datnoff et al., 1997; Correa-Victoria et al., 2001).

No Brasil, a aplicação de $800 \mathrm{~kg} \mathrm{ha}^{-1}$ de $\mathrm{SiO}_{2}$ reduziu a severidade da brusone nas panículas em 12 genótipos de arroz em experimento realizado em solo de cerrado (Prabhu et al., 2001). O controle atual da brusone nas folhas inclui o tratamento das sementes com fungicidas, com atividade sistêmica e efeito residual prolongado (Prabhu \& Filippi, 1993). Há necessidade de se buscar fontes de Si disponíveis no Brasil e que sejam eficientes no controle da brusone em arroz de várzea, para diminuir os danos ambientais e o custo dos tratamentos com produtos químicos, além de aumentar a quantidade e a qualidade dos grãos.

O objetivo deste trabalho foi estudar a eficiência de fontes de Si em relação ao tratamento das sementes com fungicida, na redução da severidade da brusone nas folhas do arroz irrigado.

\section{Material e Métodos}

O experimento foi realizado no campo, na fazenda Palmital, Município de Brazabrantes, Goiás, em área de várzea, durante os anos agrícolas 1999/2000 e 2000/2001, utilizando a cultivar Metica-1. O solo Gley Húmico apresentava as seguintes características químicas: $\mathrm{pH}$ (água), 4,5; $\mathrm{em} \mathrm{mmol} \mathrm{dm}^{-3}, \mathrm{Ca}, 10,0 ; \mathrm{Mg}, 2,9$; $\mathrm{H}+\mathrm{Al}, 75,7$; e em mg dm${ }^{-3}, \mathrm{P}, 22,27 ; \mathrm{K}, 75,0 ; \mathrm{Cu}, 3,4$;
$\mathrm{Fe}, 473,0 ; \mathrm{Mn}, 35,0$ e matéria orgânica $\left(\mathrm{g} \mathrm{dm}^{-3}\right), 9,0$. $\mathrm{O}$ experimento foi repetido por dois anos no mesmo local para estudar o efeito residual da adubação silicatada no controle da brusone. Os resultados da análise química do solo, realizada no laboratório de análise de solo da Embrapa-Centro Nacional de Pesquisa de Arroz e Feijão, são apresentados na Tabela 1.

O solo foi adubado com $350 \mathrm{~kg} \mathrm{ha}^{-1}$ da fórmula NPK (5-30-15+Zn), $250 \mathrm{~kg} \mathrm{ha}^{-1}$ de sulfato de amônio e $20 \mathrm{~kg} \mathrm{ha}^{-1}$ de zinco, por ocasião do plantio dos experimentos. A adubação de cobertura foi feita aos 30 e aos 50 dias após a semeadura, com $133 \mathrm{~kg} \mathrm{ha}^{-1}$ de sulfato de amônio em cada data.

Foi utilizado o delineamento em blocos ao acaso com quatro repetições em esquema de parcelas subdivididas. As parcelas consistiram de três fontes de $\mathrm{Si}$, rocha de serpentinita (S), silicato de alto forno (SAF) e wollastonita (W) e as subparcelas, das doses $0,0,5,1,0$, 2,0 e 4,0 Mg ha ${ }^{-1}$ de escória básica de cada fonte de $\mathrm{Si}$, em combinação com os tratamentos das sementes não tratadas e das sementes tratadas com fungicida pyroquilon (200 g de i.a. por $100 \mathrm{~kg}$ de sementes).

A rocha wollastonita $(\mathrm{W})$ é um metasilicato de cálcio natural, importado, comercializado como Vansil-EW20, que apresenta a seguinte composição em porcentagem: $\mathrm{SiO}_{2}, 51,9 ; \mathrm{CaO}, 42,1 ; \mathrm{Al}_{2} \mathrm{O}_{3}, 1,82 ; \mathrm{MgO}, 1,49$; $\mathrm{Fe}_{2} \mathrm{O}_{3}, 0,34 ; \mathrm{Na}_{2} \mathrm{O}, 0,27 ; \mathrm{MnO}, 0,03$. A rocha de serpentinita (S), comercialmente conhecida como MB-4, apresenta em porcentagem: $\mathrm{SiO}_{2}, 47,96 ; \mathrm{CaO}, 2,23$; $\mathrm{P}_{2} \mathrm{O}_{5}, 1,04 ; \mathrm{MgO}, 19,14 ; \mathrm{K}_{2} \mathrm{O}, 1,20 ; \mathrm{Fe}_{2} \mathrm{O}_{3}, 6,72$; $\mathrm{Na}_{2} \mathrm{O}, 1,14 ; \mathrm{S}, 0,55 ; \mathrm{MnO}, 0,06 ; \mathrm{Cu}, 0,012 ; \mathrm{Zn}, 0,02$; $\mathrm{Co}, 0,015$. O resíduo de indústria de fósforo (SAF) apresenta em porcentagem: $\mathrm{SiO}_{2}, 42,0 ; \mathrm{CaO}, 40,0 ; \mathrm{P}_{2} \mathrm{O}_{5}, 1,0$; $\mathrm{MgO}, 9,0 ; \mathrm{K}_{2} \mathrm{O}, 0,9 ; \mathrm{Fe}_{2} \mathrm{O}_{3}, 1,6 ; \mathrm{S}, 0,9 ; \mathrm{MnO}, 2,9$; $\mathrm{Cu}, 0,03 ; \mathrm{Zn}, 0,07$; Co, 0,007; Mo, 0,01 e B, 0,015.

Os plantios foram efetuados em canteiros de $0,30 \mathrm{~m}$ de altura, 1,50 m de largura e 45,0 $\mathrm{m}$ de comprimento, revestidos com placas de cimento pré-moldadas para evitar erosão e manter os tratamentos até o segundo ano. O tamanho da subparcela foi $1,0 \mathrm{~m}^{2}$, com dez linhas de $1,0 \mathrm{~m}$ de comprimento, com $0,10 \mathrm{~m}$ entre as linhas. A densidade de semeadura foi de 80 sementes por metro linear. Uma bordadura infestante com mistura de cultivares suscetíveis foi estabelecida nos dois lados de cada bloco no sentido perpendicular às linhas, 30 dias antes da instalação do experimento. As subparcelas foram separadas com três linhas de uma cultivar moderadamente resistente à brusone (CNA 7830) e tratada com o fungicida pyroquilon ( $200 \mathrm{~g}$ de i.a. por $100 \mathrm{~kg}$ de sementes), a fim de diminuir a interferência entre as subparcelas. $\mathrm{O}$ controle de ervas daninhas foi feito por meio de capinas, sempre 
que necessário. $\mathrm{O}$ experimento foi protegido contra o ataque de pragas pela aplicação de inseticidas recomendados para a cultura.

As variáveis de avaliação incluíram a severidade da brusone nas folhas e peso de matéria seca da parte aérea (biomassa). As observações de severidade, utilizando uma escala de 10 graus de acordo com Notteghem (1981), foram realizadas aos $30(21 / 3 / 2000), 33(24 / 3 / 2000)$ e 38 (29/3/2000) dias após o plantio (DAP) em 1999/2000, e aos 25 (14/2/2001), 30 (19/2/2001), 33 (22/2/2001) e 43 (2/3/2001) DAP em 2000/2001. A área sob a curva de progresso (ASCPD) foi calculada de acordo com Shaner \& Finney (1977) com base nas três e quatro observações de severidade da brusone, no primeiro e segundo ano agrícola, respectivamente. A biomassa foi obtida pela secagem em estufa, a $60^{\circ} \mathrm{C}$ até atingir peso constante, da parte aérea das plantas retiradas em 2 metros lineares aos 50 dias após o plantio nos dois anos. As análises de variância e de regressão foram realizadas com dados da área sob curva de progresso da brusone nas folhas e biomassa, a fim de estudar o efeito dos tratamentos e as interações entre doses de $\mathrm{Si}$ e fungicida no controle de brusone nas folhas. Foram utilizadas análises de correlação e regressão linear.
A variável dependente era constituída pela biomassa e a variável independente pela ASCPD. A análise para verificar as diferenças entre os coeficientes de regressão (b) e interseção (a) foi realizada pelo teste F (Gomez \& Gomez, 1984). As porcentagens de redução de ASCPD para determinar a eficiência do tratamento das sementes com fungicida foram calculadas por meio de análise de regressão.

\section{Resultados e Discussão}

Não houve diferenças significativas quanto à ASCPD entre as fontes de Si no primeiro ano (Tabela 2). Entretanto, as diferenças foram significativas no segundo ano, indicando diferenças no efeito residual das fontes de silício. As interações entre as fontes de $\mathrm{Si}$ e doses, e entre as fontes de $\mathrm{Si}$ e tratamentos das sementes, foram significativas em ambos os anos e entre doses de Si e tratamentos das sementes, apenas em 1999/2000 (Tabela 2). A resposta do acúmulo de matéria seca em relação às fontes e doses de Si não foi significativa, diferin-

Tabela 1. Análise química de solo $(0-20 \mathrm{~cm})$ sob os tratamentos com as fontes silicatadas no segundo ano agrícola. Embrapa-Centro Nacional de Pesquisa de Arroz e Feijão, 2000/2001.

\begin{tabular}{|c|c|c|c|c|c|c|c|c|c|c|c|}
\hline $\begin{array}{l}\text { Doses de } \mathrm{Si} \\
\left(\mathrm{Mg} \mathrm{ha}^{-1}\right)\end{array}$ & $\begin{array}{c}\mathrm{pH} \\
\text { água }\end{array}$ & $\begin{array}{l}\mathrm{Ca} \\
----(\mathrm{m}\end{array}$ & $\begin{array}{c}\mathrm{Mg} \\
\left.\mathrm{ol}_{\mathrm{c}} \mathrm{dm}^{-3}\right)\end{array}$ & $\mathrm{H}+\mathrm{Al}$ & $P$ & K & $\begin{array}{l}\mathrm{Cu} \\
-(\mathrm{m}\end{array}$ & $\begin{array}{l}\mathrm{Zn} \\
-3)\end{array}$ & $\mathrm{Fe}$ & Mn & $\begin{array}{c}\mathrm{MO} \\
\left(\mathrm{g} \mathrm{dm}^{-3}\right)\end{array}$ \\
\hline & \multicolumn{11}{|c|}{ Rocha serpentinita } \\
\hline 0,0 & 4,7 & 15,2 & 4,2 & 79 & 20,6 & 66 & 5,2 & 8,7 & 462 & 85 & 7 \\
\hline 0,5 & 4,7 & 14,4 & 4,0 & 71 & 20,6 & 78 & 5,1 & 7,9 & 396 & 76 & 7 \\
\hline 1,0 & 4,7 & 17,1 & 4,6 & 72 & 19,7 & 73 & 5,1 & 8,7 & 440 & 82 & 10 \\
\hline 2,0 & 4,7 & 17,1 & 4,8 & 76 & 18,1 & 67 & 4,8 & 7,5 & 407 & 82 & 9 \\
\hline \multirow[t]{2}{*}{4,0} & 4,8 & 19,8 & 6,5 & 81 & 21,5 & 80 & 4,9 & 7,7 & 462 & 84 & 10 \\
\hline & \multicolumn{11}{|c|}{ Silicato de alto forno } \\
\hline 0,0 & 4,7 & 14,4 & 4,0 & 71 & 20,4 & 73 & 5,2 & 8,1 & 429 & 85 & 7 \\
\hline 0,5 & 4,7 & 14,4 & 3,9 & 84 & 20,0 & 78 & 5,0 & 8,1 & 374 & 81 & 9 \\
\hline 1,0 & 4,9 & 18,0 & 4,3 & 80 & 17,2 & 86 & 5,0 & 7,3 & 374 & 85 & 8 \\
\hline 2,0 & 4,8 & 16,2 & 4,7 & 77 & 16,8 & 70 & 5,0 & 7,0 & 385 & 86 & 7 \\
\hline \multirow[t]{2}{*}{4,0} & 4,9 & 18,0 & 5,0 & 77 & 16,1 & 78 & 4,9 & 7,3 & 352 & 89 & 9 \\
\hline & \multicolumn{11}{|c|}{ Rocha wollastonita } \\
\hline 0,0 & 4,8 & 16,2 & 4,7 & 78 & 14,3 & 67 & 5,0 & 6,8 & 374 & 85 & 9 \\
\hline 0,5 & 4,8 & 15,3 & 3,9 & 78 & 15,5 & 66 & 5,1 & 6,9 & 319 & 88 & 8 \\
\hline 1,0 & 4,7 & 15,3 & 3,5 & 72 & 15,5 & 67 & 4,8 & 6,8 & 330 & 85 & 7 \\
\hline 2,0 & 5,0 & 18,9 & 4,3 & 71 & 16,1 & 73 & 5,1 & 6,7 & 341 & 88 & 9 \\
\hline 4,0 & 5,0 & 27,9 & 4,3 & 73 & 16,1 & 58 & 5,2 & 7,2 & 352 & 88 & 9 \\
\hline
\end{tabular}


do dos resultados obtidos por Winslow (1992), que encontrou resposta do arroz ao $\mathrm{Si}\left(18,7 \mathrm{~g} \mathrm{~m}^{-2}\right) \mathrm{em}$ relação ao acréscimo da massa vegetal, que em alguns casos foi de até $120 \%$. A biomassa aumentou com o tratamento das sementes com fungicida, mas não ocorreram interações significativas. As correlações entre a severidade da brusone nas folhas nas diferentes épocas do desenvolvimento das plantas na fase vegetativa e a ASCPD foram altamente significativas (Tabela 3). Os níveis de severidade da brusone e da ASCPD foram negativamente correlacionados com a biomassa conforme relatos de Prabhu et al. (1986).

As relações entre a ASCPD e as doses das fontes de Si para wollastonita (W) $(\mathrm{r}=0,87, \mathrm{P} \leq 0,05)$ e silicato de alto forno (SAF) $(r=0,90, P \leq 0,05)$ foram lineares e negativas, no primeiro ano do experi- mento, na ausência do tratamento das sementes, indicando a eficiência de SAF no controle da brusone nas folhas (Figura 1). Entretanto, não ocorreram diferenças significativas nos valores de ' $b$ ' $\left(\mathrm{F}=0,91^{\mathrm{ns}}\right)$. Foram obtidos resultados semelhantes com o tratamento das sementes com fungicida em relação a $\mathrm{W}(\mathrm{r}=0,90, \mathrm{P} \leq 0,05)$ e $\mathrm{SAF}(\mathrm{r}=0,92$, $\mathrm{P} \leq 0,05$ ) (Figura 1). As diferenças entre as inclinações das curvas não foram significativas, indicando que a redução da severidade da brusone com o aumento das doses é semelhante para os dois produtos. Esta relação entre o aumento da adubação silicatada com a redução das doenças do arroz, principalmente a brusone, foi relatada por Datnoff et al. (1991, 1992, 1997), Osuma-Canizales et al. (1991), Winslow (1992), Zambolim \& Ventura (1993) e Korndörfer et al. (1999). Por outro lado, a relação

Tabela 2. Análise de variância da área sob a curva de progresso da doença (ASCPD) da brusone nas folhas e da biomassa, nos dois anos de avaliação de fontes de silício na cultura do arroz (cultivar Metica-1) em solo de várzea. Embrapa-Centro Nacional de Pesquisa de Arroz e Feijão, 1999/2001.

\begin{tabular}{|c|c|c|c|c|c|}
\hline \multirow[t]{2}{*}{ Fonte de variação } & \multirow[t]{2}{*}{ GL } & \multicolumn{2}{|c|}{ QM } & \multicolumn{2}{|c|}{$\mathrm{F}$} \\
\hline & & $1999 / 2000$ & $2000 / 2001$ & $1999 / 2000$ & $2000 / 2001$ \\
\hline & \multicolumn{5}{|c|}{ ASCPD } \\
\hline Repetição & 3 & $14.305,3$ & $147.948,9$ & $0,22^{\mathrm{ns}}$ & $9,28 *$ \\
\hline Fonte de silício (A) & 2 & $77.565,5$ & $189.269,3$ & $1,21^{\mathrm{ns}}$ & $11,87 * *$ \\
\hline Erro & 6 & $63.897,7$ & $15.939,4$ & - & - \\
\hline Doses (B) & 4 & $61.204,2$ & $99.959,3$ & $13,92 * *$ & $8,71 * *$ \\
\hline Interação $\mathrm{AB}$ & 8 & $25.420,2$ & $28.428,7$ & $5,50 * *$ & $2,47 *$ \\
\hline Fungicida (C) & 1 & $8.763 .169,9$ & $23.648 .994,9$ & $1.897,08 * *$ & $2.059,88 * *$ \\
\hline Interação AC & 2 & $59.195,8$ & $40.610,3$ & $12,81 * *$ & $3,53 *$ \\
\hline Interação BC & 4 & $26.933,9$ & $5.123,8$ & $5,83 * *$ & $0,44^{\mathrm{ns}}$ \\
\hline Interação $\mathrm{ABC}$ & 8 & $10.331,7$ & $12.217,9$ & $2,23 *$ & $1,77^{\mathrm{ns}}$ \\
\hline Erro & 81 & $5.132,8$ & $11.480,7$ & - & - \\
\hline \multirow[t]{2}{*}{ Total } & 119 & & & & \\
\hline & \multicolumn{5}{|c|}{ Biomassa } \\
\hline Repetição & 3 & 355,6 & $1.151,2$ & $0,78^{\mathrm{ns}}$ & $3,05^{\mathrm{ns}}$ \\
\hline Fonte de silício (A) & 2 & 613,5 & 352,9 & $1,35^{\mathrm{ns}}$ & $0,94^{\mathrm{ns}}$ \\
\hline Erro & 6 & 453,0 & 376,3 & - & - \\
\hline Doses (B) & 4 & 61,7 & 126,0 & $0,72^{\mathrm{ns}}$ & $1,91^{\mathrm{ns}}$ \\
\hline Interação AB & 8 & 101,81 & 77,3 & $1,18^{\mathrm{ns}}$ & $1,17^{\mathrm{ns}}$ \\
\hline Fungicida (C) & 1 & $16.492,4$ & $16.234,6$ & $191,6 * *$ & $245,85 * *$ \\
\hline Interação AC & 2 & 134,1 & 71,8 & $1,55^{\mathrm{ns}}$ & $1,09^{\mathrm{ns}}$ \\
\hline Interação BC & 4 & 141,8 & 21,0 & $1,65^{\mathrm{ns}}$ & $0,32^{\text {ns }}$ \\
\hline Interação ABC & 8 & 64,5 & 34,8 & $0,75^{\mathrm{ns}}$ & $0,53^{\mathrm{ns}}$ \\
\hline Erro (ABC) & 81 & 86,1 & 66,0 & - & - \\
\hline Total & 119 & & & & \\
\hline
\end{tabular}

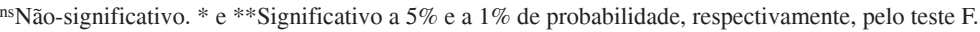


não foi significativa para rocha de serpentinita $(\mathrm{S})$ sem tratamento $\left(r=0,35^{\text {ns }}\right)$ e com tratamento de sementes $\left(r=0,14^{\mathrm{ns}}\right)$.

No segundo ano, as relações entre ASCPD e doses das fontes de $\mathrm{W}$ e SAF foram negativas e significativas, com ou sem tratamento das sementes com o fungicida, como no primeiro ano do experimento (Figura 2). Entretanto, os valores de ASCPD foram maiores sem aplicação de Si no segundo ano, indicando alta pressão da doença. Nestas condições, não se obteve diferenças significativas entre as inclinações das curvas, $\operatorname{sem}\left(\mathrm{F}=0,52^{\mathrm{ns}}\right)$ ou com $\left(\mathrm{F}=3,49^{\mathrm{ns}}\right)$ uso do fungicida no tratamento das sementes. Por sua vez, as diferenças entre valores de 'a' (Figura 2) podem ser explicadas pelos níveis da brusone nas parcelas, como foi evidenciado pela análise de

Tabela 3. Correlações entre as variáveis e a área sob a curva de progresso da doença (ASCPD) da brusone nas folhas e a massa seca da parte aérea (biomassa), nos dois anos de avaliação de fontes de silício na cultura do arroz (cultivar Metica-1) em solo de várzea. Embrapa-Centro Nacional de Pesquisa de Arroz e Feijão, 1999/2001(1).

\begin{tabular}{lccc}
\hline Variáveis & Data da avaliação & \multicolumn{2}{c}{$1999 / 2000$} \\
\cline { 3 - 4 } & & ASCPD & Biomassa \\
\hline Severidade da brusone nas folhas & $21 / 3 / 2000$ & $0,98^{* *}$ & $-0,77^{* *}$ \\
Severidade da brusone nas folhas & $24 / 3 / 2000$ & $0,95^{* *}$ & $-0,74^{* *}$ \\
Severidade da brusone nas folhas & $29 / 3 / 2000$ & $0,93^{* *}$ & $-0,74^{* *}$ \\
ASCPD & & & $-0,78^{* *}$ \\
\hline & & & $2000 / 2001$ \\
Severidade da brusone nas folhas & $14 / 2 / 2001$ & $0,77^{* *}$ & $-0,55^{* *}$ \\
Severidade da brusone nas folhas & $19 / 2 / 2001$ & $0,92^{* *}$ & $-0,70^{* *}$ \\
Severidade da brusone nas folhas & $22 / 2 / 2001$ & $0,95^{* *}$ & $-0,74^{* *}$ \\
Severidade da brusone nas folhas & $2 / 3 / 2001$ & $0,83^{* *}$ & $-0,79^{* *}$ \\
ASCPD & & & $-0,79^{* *}$ \\
\hline
\end{tabular}

${ }^{(1)}$ Foram realizadas 120 observações em cada ano; em 1999/2000, as datas de avaliação corresponderam a 30, 33 e 38 dias após o plantio, respectivamente, e em 2000/2001, corresponderam a 25, 30, 33 e 43 dias após plantio, respectivamente. **Significativo a $1 \%$ de probabilidade.
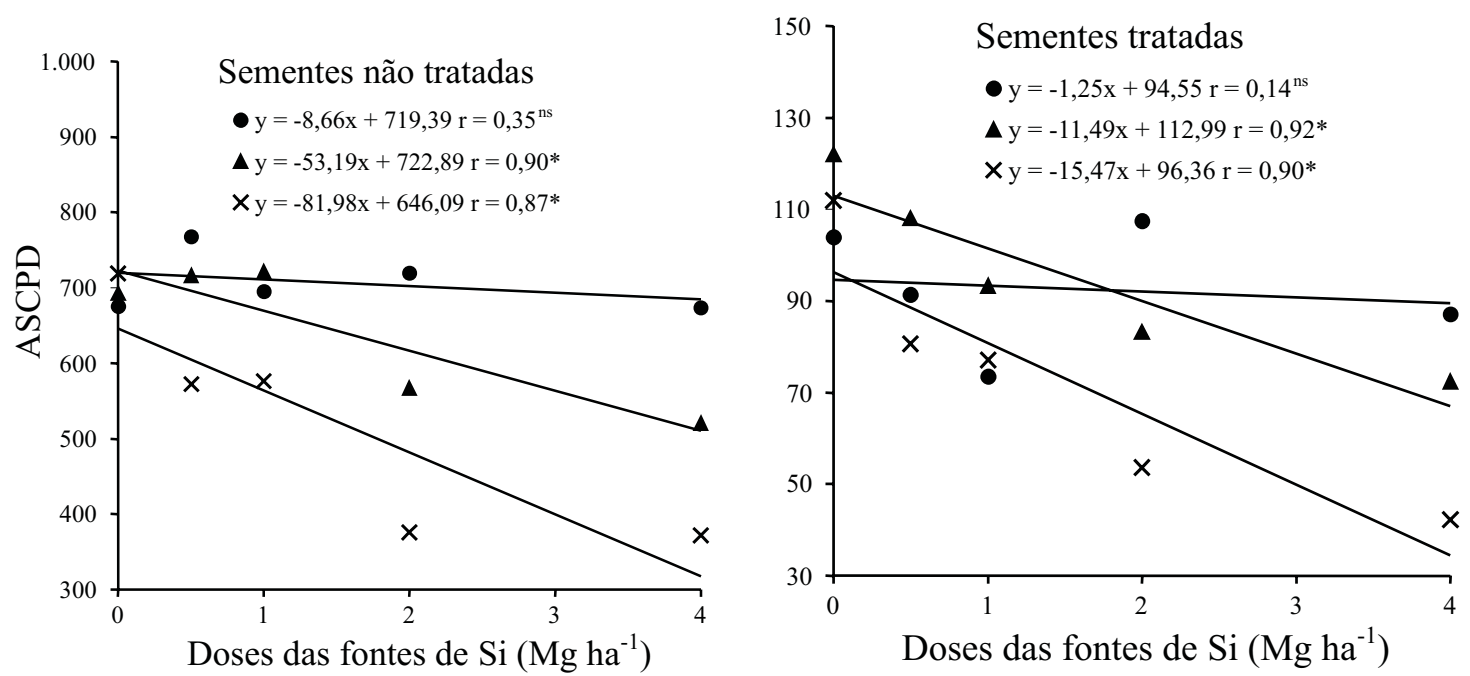

Figura 1. Efeito das fontes de silício (rocha serpentinita, $\bullet$; silicato de alto forno, $\mathbf{\Lambda}$; e rocha wollastonita,X) na severidade da brusone nas folhas, obtida pela área sob a curva de progresso da doença (ASCPD), em arroz cultivar Metica-1, cujas sementes haviam sido tratadas ou não com o fungicida pyroquilon (200 g de i.a. por $100 \mathrm{~kg}$ de sementes), em solo de várzea. ${ }^{\text {ns }}$ ão-significativo. *Significativo a $5 \%$ de probabilidade. 

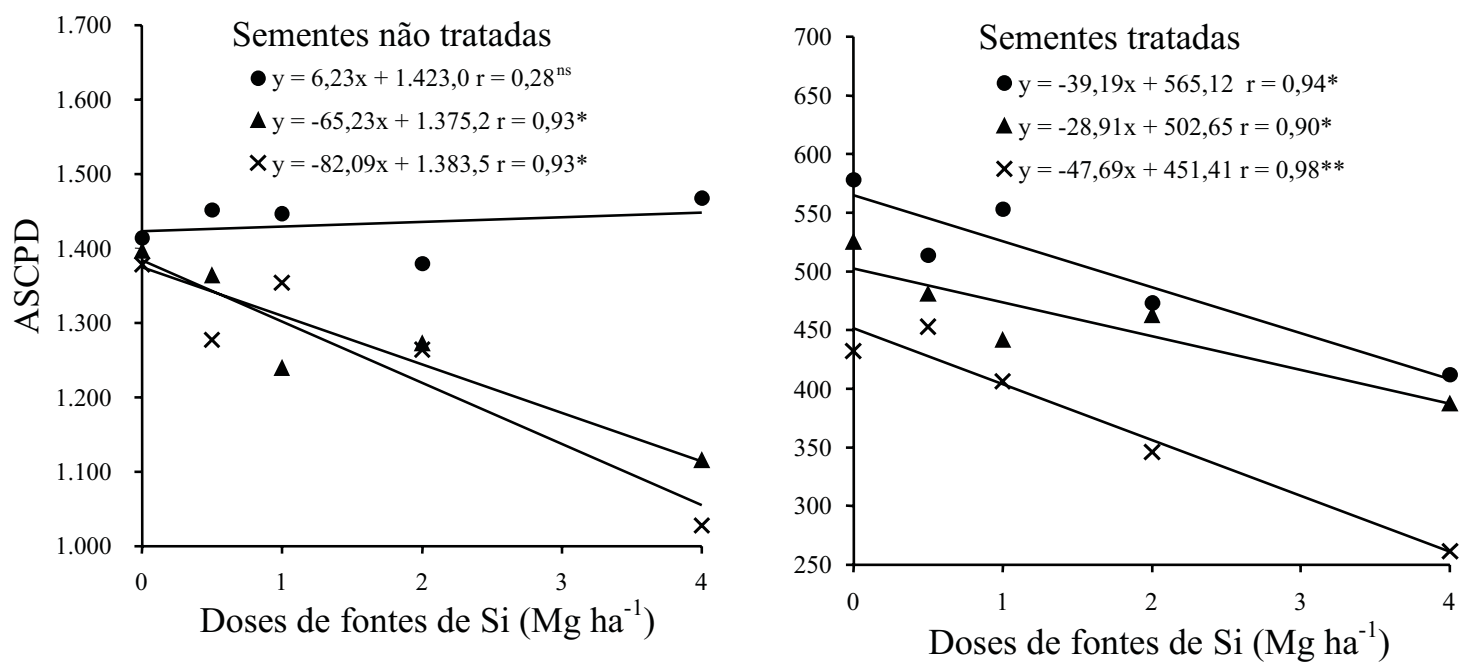

Figura 2. Efeito das fontes de silício (rocha serpentinita, $\bullet$; silicato de alto forno, $\mathbf{\Delta}$; e rocha wollastonita, $\mathbf{X}$ ) na severidade da brusone nas folhas, obtida pela área sob a curva de progresso da doença (ASCPD), em arroz cultivar Metica-1, cujas sementes haviam sido tratadas ou não com o fungicida pyroquilon (200 g de i.a. por $100 \mathrm{~kg}$ de sementes), em solo de várzea. ${ }^{\text {ns }}$ ão-significativo. * e ${ }^{* *}$ Significativo a $5 \%$ e a $1 \%$ de probabilidade, respectivamente.

variância (Tabela 2). A brusone diminuiu significativamente $(r=0,94, \mathrm{P} \leq 0,01)$ com o aumento das doses de rocha de serpentinita na presença do tratamento das sementes. Estes resultados mostraram o efeito residual da aplicação das fontes de silício. Resultados semelhantes foram obtidos por Datnoff et al. (1997) e Correa-Vitoria et al. (2001).

Conforme as equações de regressão obtidas em 1999/2000, na ausência da fonte de $\mathrm{Si}$, as porcentagens de controle foram $85,0 \%$ e $84,4 \%$, mas, com aplicação de $4 \mathrm{Mg} \mathrm{ha}^{-1}$ de W e SAF, a eficiência de controle aumentou para $89,2 \%$ e $86,9 \%$, respectivamente. No segundo ano, mesmo sob alta pressão de doença, as porcentagens de redução da ASCPD com o tratamento das sementes, foram $67,4 \%$ e $63,4 \%$, e, com aplicação de $4 \mathrm{Mg} \mathrm{ha}^{-1}$ de W e SAF, a eficiência de controle aumentou para $75,3 \%$ e $65,3 \%$, respectivamente, indicando um possível efeito sinergístico. Esta eficiência do tratamento das sementes com o fungicida pyroquilon é o que o torna uma prática rotineira usada pelos produtores (Prabhu \& Filippi, 1993), apesar do alto custo envolvido em sua utilização, quando comparado aos demais fungicidas no mercado. A redução da ASCPD com adubação silicatada de $4 \mathrm{Mg} \mathrm{ha}^{-1}$, sem tratamento das sementes, no primeiro e segundo anos foram $29,4 \%$ e $19,0 \%$ para SAF e $50,7 \%$ e $23,9 \%$ para W, respectivamente (Figuras 1 e 2).

Apesar da maior quantidade de Si presente na rocha de serpentinita, esta fonte apresentou menor efeito do que o silicato de alto forno, possivelmente em razão das diferenças na granulometria, composição química, solubilidade e dos processos de obtenção destes materiais. A fonte SAF é processada em altas temperaturas e apresenta maior conteúdo de cálcio. Takahashi (1981) demonstrou que a maior disponibilidade do Si proveniente das escórias é encontrada nos materiais com processo de esfriamento mais lento, menor granulometria e maior conteúdo de cálcio.

A cultivar Metica-1 é altamente suscetível à brusone. São recomendáveis estudos mais detalhados com cultivares moderadamente suscetíveis, pois a resposta à aplicação do Si quanto ao controle dessa doença poderá ser maior.

\section{Conclusões}

1. Existem fontes de silício disponíveis no Brasil que reduzem a brusone nas folhas do arroz. 
2. A brusone nas folhas do arroz diminui com o aumento da dose de silício.

3. A eficiência do tratamento das sementes com fungicida no controle da brusone nas folhas do arroz aumenta com a aplicação de silício.

\section{Referências}

BARBOSA FILHO, M. P.; SNYDER, G. H.; FAGERIA, N. K.; DATNOFF, L. E.; SILVA, O. F. da. Silicato de cálcio como fonte de silício para o arroz de sequeiro. Revista Brasileira de Ciência do Solo, Viçosa, MG, v. 25, n. 2, p. 325-330, 2001.

CORREA-VICTORIA, F. J.; DATNOFF, L. E.; OKADA, K.; FRIESEN, D. K.; SANZ, J. I.; SNYDER, G. H. Effects of silicon fertilization on disease development and yields of rice in Colombia. In: DATNOFF, L. E.; SNYDER, G. H.; KORNDÖRFER, G. H. (Ed.). Silicon in agriculture. Amsterdam: Elsevier, 2001. p. 313-322.

DATNOFF, L. E.; DEREN, C. W.; SNYDER, G. H. Silicon fertilization for disease management of rice in Florida. Crop Protection, London, v. 16, n. 6, p. 525-531, 1997.

DATNOFF, L. E.; RAID, R. N.; SNYDER, G. H.; JONES, D. B. Effect of calcium silicate on blast and brown spot intensities and yields of rice. Plant Disease, St. Paul, v. 75, n. 7, p. 729-732, 1991.

DATNOFF, L. E.; SNYDER, G. H.; DEREN, C. W. Influence of silicon fertilizer grades on blast and brown spot development and on rice yields. Plant Disease, St. Paul, v. 76, n. 10, p. 1011-1013, 1992.

GOMEZ, K. A.; GOMEZ, A. A. Statistical procedures for agricultural research. 2nd ed. Singapore: J. Wiley, 1984. $680 \mathrm{p}$.

GOTO, K. Estimating losses from rice blast in Japan. In: GOTO, K. The rice blast disease. Baltimore: Johns Hopkins Press, 1965. p. 195-202.

KORNDÖRFER, G. K.; DATNOFF, L. E.; CORRÊA, G. F. Influence of silicon on grain discoloration and upland rice grown on four savanna soils of Brazil. Journal of Plant Nutrition, New York, v. 22, n. 1, p. 93-102, 1999.

NOTTEGHEM, J. L. Cooperative experiment on horizontal resistance to rice blast. In: INTERNATIONAL RICE RESEARCH INSTITUTE (Los Baños, Filipinas). Blast and upland rice: report and recommendations from the meeting for international collaboration in upland rice improvement. Los Baños, 1981. p. 43-51.
OSUMA-CANIZALES, F. J.; DEDATTA, S. K.; BONMAN, J. M. Nitrogen form and silicon nutrition effects on resistance to blast disease of rice. Plant and Soil, The Hague, v. 135, p. 223-231, 1991.

PRABHU, A. S.; FILIPPI, M. C. Seed treatment with pyroquilon for the control of leaf blast in Brazilian upland rice. International Journal of Pest Management, Hampshire, v. 39, p. 347-353, 1993.

PRABHU, A. S.; BARBOSA FILHO, M. P.; FILIPPI, M. C.; DATNOFF, L. E.; SNYDER, G. H. Silicon from disease control perspective in Brazil. In: DATNOFF, L. E.; SNYDER, G. H.; KORNDÖRFER, G. H. Silicon in agriculture. Amsterdam: Elsevier, 2001. p. 293-311.

PRABHU, A. S.; FARIA, J. C.; CARVALHO, J. R. P. Efeito da brusone sobre a matéria seca, produção de grãos e seus componentes em arroz de sequeiro. Pesquisa Agropecuária Brasileira, Brasília, v. 21, n. 5, p. 495-500, maio 1986.

PRABHU, A. S.; FARIA, J. C.; ZIMMERMANN, F. J. P. Comparative yield loss estimates due to blast in some upland rice cultivars. Fitopatologia Brasileira, v. 14, n. 3/4, p. 227-232, out./dez. 1989.

SAVANT, N. K.; SNYDER, G. H.; DATNOFF, L. E. Silicon management and sustainable rice production. Advances in Agronomy, New York, v. 58, p. 151-199, 1997.

SHANER, G.; FINNEY, R. E. The effect of nitrogen fertilization on the expression of slow mildewing resistance in Knox wheat. Phytopathology, St. Paul, v. 67, p. 10511056, 1977.

SUZUKI, N. Nature of resistance to blast. In: GOTO, K. The rice blast disease. Baltimore: Johns Hopkins Press, 1965. p. 277-302.

TAKAHASHI, K. Effects of slags on the growth and the silicon uptake by rice plants and the available silicates in paddy soils. Bulletin of the Shikoku Agricultural Experimental Station, Kagawa, n. 38, p. 75-114, 1981.

WINSLOW, M. D. Silicon disease resistance and yield of rice genotypes under upland cultural conditions. Crop Science, Madison, v. 32, p. 1208-1213, 1992.

ZAMBOLIM, L.; VENTURA, J. A. Resistência a doenças induzida pela nutrição mineral das plantas. Revisão Anual de Patologia de Plantas, Passo Fundo, v. 1, p. $275-$ 318, 1993. 